\title{
Re-examining the correlation of complex solar type III radio bursts and solar energetic particles
}

\author{
R. J. MacDowall ${ }^{1}$, I. G. Richardson ${ }^{2}$, R. A. Hess ${ }^{3}$, and G. Thejappa ${ }^{4}$ \\ ${ }^{1}$ NASA Goddard Space Flight Center, Code 695, Greenbelt, MD 20771, USA \\ email: robert.macdowall@nasa.gov \\ ${ }^{2}$ Code 661, NASA Goddard Space Flight Center, Greenbelt, MD 20771, and \\ CRESST and Department of Astronomy, University of Maryland, College Park, MD 20742 \\ email: ian.g.richardson@nasa.gov \\ ${ }^{3}$ NASA Goddard Space Flight Center, Code 695, Greenbelt, MD 20771, USA, and \\ Wyle Information Systems Group, McLean, VA 22102 \\ email: roger.hess@nasa.gov \\ ${ }^{4}$ NASA Goddard Space Flight Center, Code 695, Greenbelt, MD 20771, USA, and \\ Department of Astronomy, University of Maryland, College Park, MD 20742 \\ email: thejappa.golla@nasa.gov
}

\begin{abstract}
Interplanetary radio observations provide important information on particle acceleration processes at the Sun and propagation of the accelerated particles in the solar wind. Cane et al. (2002) have drawn attention to a class of prominent radio bursts that accompany $>20$ $\mathrm{MeV}$ solar proton events. They call these bursts 'type III-L' because: they are fast drifting (like normal type III bursts associated with electrons accelerated at impulsive solar flares); they are Long-lasting compared to normal type III bursts; they occur Late compared to the onset of the related solar event; and, they commence at Lower frequencies $(\sim 100 \mathrm{MHz})$ than normal type III bursts, suggesting that they originate higher in the corona at $\sim 0.5 \mathrm{R}_{s}$ above the Sun. We report on an analysis of the correlated radio and SEP events during 1996-2006 using the Wind Waves and near-Earth SEP data sets, and discuss whether the characteristics of the complex type III bursts (at less than $14 \mathrm{MHz}$ ) will permit them to serve as proxies for SEP event occurrence and intensity.
\end{abstract}

Keywords. acceleration of particles, shock waves, Sun: radio radiation, Sun: coronal mass ejections (CMEs), Sun: flares

\section{Introduction}

Solar radio burst observations provide valuable information about particle acceleration in the corona and inner heliosphere. Type III bursts are radio emissions whose exciter electrons (of energies 1-10 keV) propagate rapidly from a solar flare source outwards along open magnetic field lines. Type II solar bursts result from electrons accelerated at coronal mass ejection (CME)-driven shocks or blast wave shocks in the corona. For both types of solar radio event, the emission mechanism is a plasma process where electron beam energy is converted to a plasma wave intermediary, which is then converted to a propagating electromagnetic wave.

Cane et al. (2002) defined a special class of type III bursts, based on examination of radio data from metric to kilometric wavelengths, that were found to accompany $>20 \mathrm{MeV}$ solar proton events. These bursts were Long-lasting compared to normal type III bursts; Late compared to the onset of the related solar event; and commenced at Lower frequencies $(\sim 100 \mathrm{MHz})$ than normal type III bursts (suggesting that they 


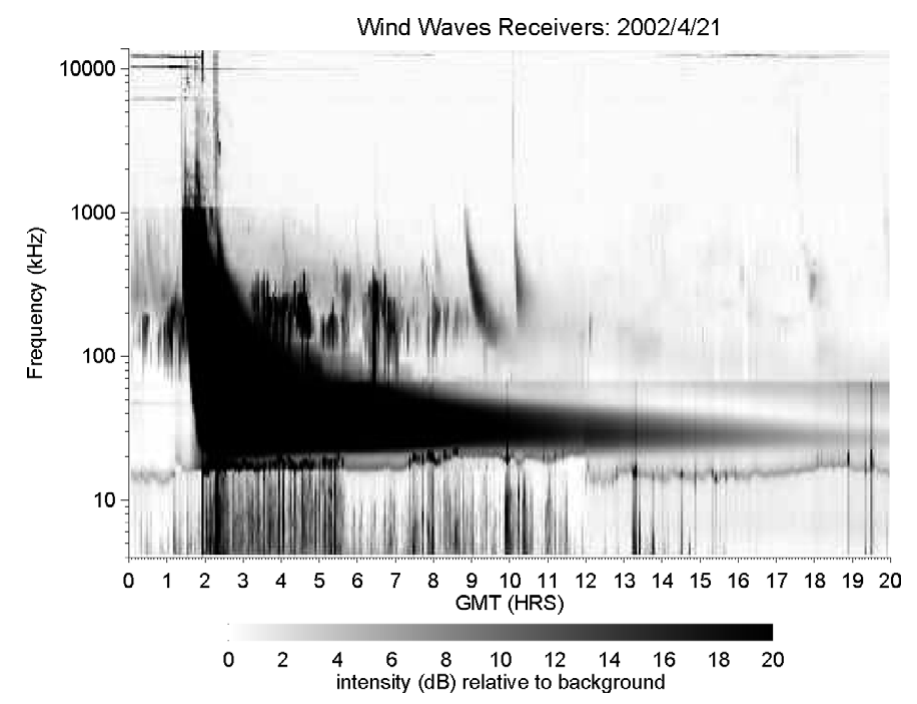

Figure 1. Twenty hours of Wind Waves data (from the RAD2, RAD1, and TNR receivers) on $2002 / 04 / 21$. The large event can be described as a type III-L burst. Note the long durations at $1 \mathrm{MHz}$ and $100 \mathrm{kHz}$ and the complexity above $1 \mathrm{MHz}$ compared to a typical, intense type III burst (see Figure 2).

originate higher in the corona, at $\sim 0.5$ Rs above the solar surface). Figure 1 presents an example of such a burst, observed by the Wind Waves instrument (Bougeret et al. (1995)).

Bursts like that in Figure 1, notable for their long-duration and complexity above 1 $\mathrm{MHz}$ and their high intensity, especially at frequencies below $100 \mathrm{kHz}$, have been studied previously by a number of authors. Cane et al. (1981) proposed that the exciter electrons were accelerated by CME-driven shocks; Kahler et al. (1986) and Dulk et al. (2000) reached similar conclusions. MacDowall et al. (1987) and Kundu et al. (1990) examined the events in the framework of shock association, rather than shock acceleration. Klein et al. (1997) and Reiner et al. (2000) pointed to correlations with GHz emissions lower in the corona to argue that the source electrons were not accelerated at shocks. Cane et al. (2002) suggested that type III-L bursts were produced by electrons accelerated by reconnection behind fast CMEs.

MacDowall et al. (2003) confirmed that bursts with type III-L characteristics are statistically associated with intense SEP (proton) events. They examined durations, intensities, and other characteristics of hectometric type III radio bursts associated with intense SEP events and compared them to several groups of control particle events. They concluded that simple criteria, based on hectometric data alone, can identify the majority $(\sim 80 \%)$ of type III-L radio bursts associated with $>20 \mathrm{MeV}$ SEP proton events, while excluding almost $100 \%$ of the control events. In this paper we examine a concise set of parameters for identifying a type III-L burst and its likely association with an SEP event.

\section{Observations}

Energetic $(>20 \mathrm{MeV})$ proton data from instruments on various near-Earth spacecraft, including IMP-8 and SOHO, are used to construct a complete list of solar energetic particle (SEP) events during 1996-2006 that are dispersive and above instrumental background 


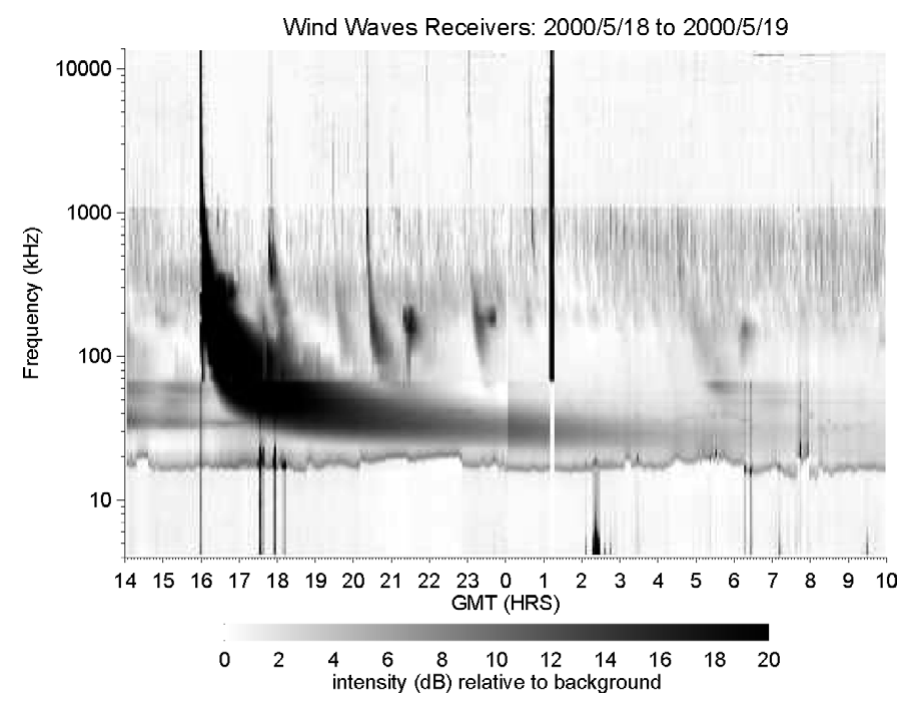

Figure 2. A classical type III burst starting on 2000/05/08. Note the much shorter duration and the simple appearance above $1 \mathrm{MHz}$. This burst is more intense than the burst in Figure 1 at $\sim 1 \mathrm{MHz}$, as indicated by the saturation-induced intermodulation seen at 16:00 below $\sim 200$ $\mathrm{kHz}$.

levels (a few events may have been missed due to data gaps or high backgrounds from preceding events). We also use data from the Wind Waves receivers, specifically, RAD2 (1-14 $\mathrm{MHz}$ ), RAD1 (52-1000 kHz), and Thermal Noise Receiver (TNR) (4-64 kHz), as shown in Figure 1, to examine the properties of the radio bursts associated with three groups of proton events. At $\sim 25 \mathrm{MeV}$ during the first 12 hours of an event, these three groups have maximum intensities in well-separated bins defined by (1) $0.0009-0.001$ protons/ $\left(\mathrm{cm}^{2}\right.$-sec-ster-MeV), (2) $0.1-0.2$ protons/ ( $\mathrm{cm}^{2}$-sec-ster-MeV), and (3) > 1 protons/ $\left(\mathrm{cm}^{2}\right.$-sec-ster-MeV). We also selected by visual inspection a control group of intense type III bursts that are not associated with any significant level of SEP activity at or above $25 \mathrm{MeV}$; an example is shown in Figure 2.

\section{Results}

In Figure 3, the radio burst durations at $1 \mathrm{MHz}\left(\sim 10 \mathrm{R}_{s}\right)$ determined from RAD1 data are plotted for the 4 groups. Although there is some overlap, the range of burst durations clearly increases with SEP event intensity. Average durations (diamonds) increase from 17 to 25 to 38 minutes for groups (1) to (3). Around a third of the events in group (3) are bursts exceeding 50 minutes, which are not observed for groups (1) or (2). Furthermore, all events in group (3) have durations exceeding $\sim 20$ minutes. In contrast, the control bursts clearly have shorter durations $(\leqslant 20 \mathrm{~min})$ than almost all of the events associated with SEPs, with a mean of $\sim 12$ minutes and a smaller spread in duration. Figure 2 shows a similar pattern for the RAD1 durations at $100 \mathrm{kHz}$ except that the durations of the control events now overlap those of the weaker SEP events. Note that the typical durations are longer for all of the groups than at $1 \mathrm{MHz}$, as is evident for the example bursts in Figures 1 and 2.

Next, we examine how the complexity of the radio burst varies with SEP event intensity. To quantify complexity we use four frequencies in the Waves RAD2 range: 13.825 (RAD2 channel 255), 6.875 (116), 3.475 (48), 1.725 (13) MHz. Radio intensity data for 

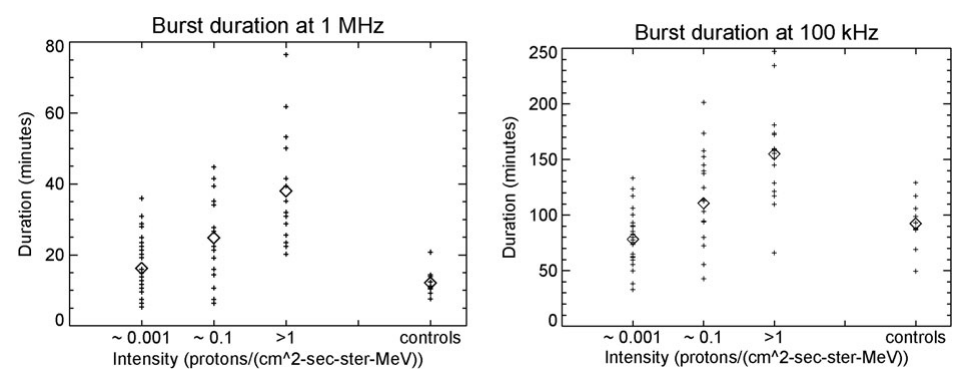

Figure 3. (Left) RAD1 burst durations at $1 \mathrm{MHz}$ for three groups of SEP events with increasing proton intensity at $\sim 25 \mathrm{MeV}$, and a control group of bursts without SEP events. Diamonds show the mean value for each group. (Right) Same format as at left for RAD1 $100 \mathrm{kHz}$ data.

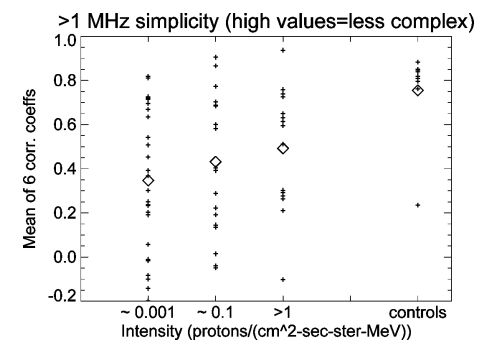

Figure 4. Radio burst simplicity factor as calculated for the 3 groups of SEP events and the control events. See description in text.

pairs of these 4 frequencies ( 6 pairs in all) are cross correlated and the 6 values are averaged. The result may be called a 'simplicity factor' because the correlation coefficients are larger for simple bursts than for more complicated bursts. The simplicity factor (SF), plotted in Figure 4, has values close to unity for similar burst profiles at the 4 frequencies, with lower values indicating profiles of greater complexity. With one exception, the control events all have simple profiles $(\mathrm{SF} \sim 0.8)$, whereas bursts associated with SEP events are more complex with a wide range of SF that shows little variation with SEP event intensity. This is consistent with the complexity previously identified as characteristic of type III-L events. Typically, the complex profiles correspond to multiple components that are seen above $1 \mathrm{MHz}$ (see Figure 1). Such components were identified by eye and counted at $2 \mathrm{MHz}$ in the analysis of MacDowall et al. (1987).

\section{Discussion}

The present study of a fraction of the events in the complete SEP event list indicates that duration ( $\sim 100 \mathrm{kHz}-1 \mathrm{MHz}$ ) and complexity $(1-10 \mathrm{MHz}$ ) of the radio bursts associated with intense $\left(>1.0 /\left(\mathrm{cm}^{2} \mathrm{~s}\right.\right.$ sr MeV $) 25 \mathrm{MeV}$ proton events are almost always greater than for the control events. For weaker SEP events, the burst duration generally decreases with proton intensity and there is increasing overlap with the control events, suggesting that it would be difficult to associate all SEP events with a distinct class of type III radio bursts. Nevertheless, long burst duration and complexity at frequencies above $1 \mathrm{MHz}$ appear to reliably select radio events that are associated with intense SEP events. This association may warrant consideration of the radio bursts as a space weather predictor. 
There are several outstanding questions which we will discuss in a future work, including: (a) What are the causes of the long duration and complexity of type III-L bursts? (b) Do type III-L bursts suggest that some flare (proton) particles contribute to SEP events? (c) How useful a role can type III-L bursts play in SEP space weather prediction?

Comparison of the complete SEP event list with the Waves radio data will permit us to better investigate the statistics of parameters defining a class of 'type III-L bursts'.

\section{Acknowledgements}

The Wind Waves investigation is a collaboration of NASA Goddard Space Flight Center (GSFC), the Observatoire de Paris-Meudon, and the University of Minnesota. Energetic particle data in this study were provided by the GSFC instrument on IMP8, the ERNE and COSTEP instruments on SOHO, and EPAM instrument on ACE. Observations from these instruments are available from the Space Physics Data Facility (http://spdf.gsfc.nasa.gov/).

\section{References}

Bougeret, J. L., et al. 1995, Space Sci. Rev., 71, 231

Cane, H. V., Stone, R. G., Fainberg, J., Stewart, R. T., Steinberg, J.-L., \& Hoang, S. 1981, Geophys. Res. Lett., 8, 1285

Cane, H. V., Erickson, W. C., \& Prestage, N. P. 2002, J. Geophys. Res., 107(A10), 1315, doi:10.1029/2001JA000320

Dulk, G. A., Leblanc Y., Bastian, T. S., \& Bougeret, J. L. 2000, J. Geophys. Res., 105, 27,343

Kahler, S. W., Cliver, E. W., \& Cane, H. V. 1986, Adv. Space Res., 6, 319

Klein, K.-L., Aurass, H., Soru-Escaut, I., \& Kalman, B. 1997, A\&A, 320, 612 (erratum 322, 1027)

Kundu, M. R., MacDowall, R. J., \& Stone, R. G. 1990, Ap\&SSS, 165, 101

MacDowall, R. J., Kundu, M. R., \& Stone, R. G. 1987 Solar Phys., 111, 397

MacDowall, R.J., Lara, A., Manoharan, P. K., Nitta, N. V., Rosas, A. M., \& Bougeret, J. L. 2003, Geophys. Res. Lett., 30, 12, 8018, doi:10.1029/2002GL016624

Reiner, M. J., Karlicky, J. M., Jiricka, K., Aurass, H., Mann, G., \& Kaiser, M. L. 2000, Astrophys. J., 530,1049

\section{Discussion}

SPANGLER: You have plotted your spectra as power level in db above background. This has problems if the background depends on frequency, and in any case, is physically uninterpretable. I would recommend plotting spectra as Janskys or antenna temperature as a function of frequency.

MACDOWALL: We can and often do that; however, given 3 instruments (here) with quite different backgrounds the result may not look the best. Furthermore, given the frequencydependent backgrounds such events can be seen more easily using relative backgrounds.

Roth: Fluxes of type III exciting electrons are often related to enhanced $\mathrm{MeV}$ heavy ions or $\mathrm{He}^{3}$. Does it apply to these complex type III events?

MACDOWALL: We haven't looked at this for these events. I recall that, for the 2002 CDAW on SEPs, there did not seem to be a good correlation between heavy ion events and the type III-l events. 
Gopalswamy: You didn't say much about CMEs. Have you looked at the evolution of magnetic arcades behind the CME to determine how the time scale compares with the radio burst duration?

MacDowall: Not yet. As I said, Dr. Cane's hypothesis was that the type III-L bursts electrons are accelerated in reconnection fields behind the CME. We should look at the arcade evolution for time correlation. 